\title{
eLetter to the Editor: Don't Believe Everything You Read: Perspectives from Your New Pain Physician
}

\section{TO THE EDITOR:}

A CNN article was recently published titled "The More Opioids Doctors Prescribe, the More Money They Make" (1). The article opens with "As tens of thousands of Americans die from prescription overdoses each year, an exclusive analysis by CNN and researchers at Harvard University found that opioid manufacturers are paying physicians huge sums of money - and the more opioids a doctor prescribes, the more money he or she makes." After arriving at the end of this sentence, it was clear this was not going to paint our field in a positive light. After reading the article to gain a thorough perspective of the authors', and perhaps the general public's, viewpoint, we felt compelled to vocalize our thoughts with hopes to educate readers of where the new generation of physicians, who may be prescribing opioids, approach this topic.

As anesthesiologists, we receive extensive training on the pharmacology, pharmokinetics, and pharmodynamics of opioids, how they interact with the human body, and importantly their adverse effects, including tolerance and addiction. In addition to our core training in Anesthesiology, we have embarked on an additional year of subspecialty training in interventional pain medicine in order to treat chronic pain utilizing a truly multimodal approach. An often over looked fact, is that the vast majority of opioid prescribers are not pain physician specialists.

As physicians training in the subspecialty of pain medicine, we see hundreds of patients in the pain clinic who take opioids on a regular basis and, yes, we do write prescriptions for some of them. That is where a definite line in the sand is drawn with regards to this article. The authors correctly highlight the inexcusable actions of a small number of "bad apple" pain physicians, but incorrectly make the mistake of generalizing such motives to the larger group of "opioid prescribers." They then further extrapolate the conclusions by intertwining opioid prescribing and monetary gain, taking readers to the incorrect, yet unavoidable conclusion, that physicians as a whole are prescribing these dangerous medications with ulterior, financial motives. As Harvard physicians ourselves, we are now a few months away from entering the work force. We have been trained in approaching pain through a complete bio-psycho-social approach and target its cause utilizing our multimodal armamentarium, which is comprised of various categories of medications. There absolutely is a place for opioid medication in pain management as long as it is used for the appropriate patient and prescribed in a safe manner, in accordance with standardized guidelines. We do not want to see the pendulum swing too far in the opposite direction, where patients who actually need these medications are denied it and their pain inappropriately managed. In our clinic, we practice an opioid sparing approach for the majority of patients, but of course exceptions need to be made and there is no compensation for this.

Unfortunately, the older generation of physicians were not equipped with the knowledge that we have today, due to major strides in research, on the effects of opioids in the long term. As a result, we have found ourselves in the midst of our current opioid epidemic. For decades, multiple generations of patients have been exposed to high dose opioids as a result of "common practice." These patients are the ones expecting to continue these medications, who now sit across from us, and instead of wanting to hear our rationale for why they should come off opioids, which includes innumerable side effects, such as respiratory depression, constipation, opioid induced hyperalgesia, and tolerance, they just want the pill that works to reduce their pain. As newly trained specialists, we understand the data from recently published randomized controlled trials, such as the SPACE trial, published this year in JAMA, whose results concluded that treatment with opioids was not superior to treatment with nonopioid medications for improving pain-related function over 12 months, thus the study does not support initiation of opioid therapy for moderate to severe chronic back pain or hip or knee osteoarthritis pain (2), but unfortunately, the addictive properties of these medications 
make it a difficult beast to tackle. We get too many patients who "have tried everything and nothing works except oxycodone, and all I want to be able to do is get out of bed in the morning. Please help." This is where physicians enter the dreaded damned if you do and damned if you don't scenario.

The article insinuates that physicians who prescribe opioids all do so for monetary gain. This further propagates the public's misconception when it comes to doctors, it is only about personal financial gain and overpayment. This article will unfortunately only add fuel to that long-burning fire, now with a new twist - supposed motivation to push dangerous medications for personal financial gains. What a majority of the public does not know is that, most physicians, including us, have over $\$ 300,000$ in student loans with interest accruing on that principal every day. In addition, the majority of the public is probably unaware that physician compensation accounts for roughly only eight percent of the total annual healthcare costs in the United States, which of course is a very small percentage of the total cost (3). Finally, the public must be aware that many strides have been made in the field of pain management over the past 2 decades that do not rely on opioids. Physicians, researchers, and industry have collaborated to produce multiple new and safer options for patients. The unfortunate reality is that legislators continue to allow third party payers to limit access to these medications and procedures. If we want to solve the opioid epidemic, we need to ensure access to nonopioid pain care and fast track nonopioid options through the Food and Drug Administration (FDA).

Although we commend the authors for bringing to light the bad practices of a few physicians, the title they chose for their article and the way they framed their introduction unfortunately leads readers down the undeniable, yet absolutely incorrect, path that the majority of physicians still believe opioids are a major part of treating chronic pain and further fuels the continued misconception that physicians are motivated by monetary gains at the expense of their patients' wellbeing. As anesthesiologists who will soon be seeing our own patients in the pain clinic, we want our future patients to know that opioids, in most circumstances, have a minor place in chronic pain management, but if they are to be prescribed, we hope it will be done in a safe, diligent manner. We hope the actions of some are not taken to represent us all. The practice of medicine requires countless years of training and hard work and is centered around our core Hippocratic oath: to do no harm.

Omar Viswanath, MD

Anesthesiologist/Interventional Pain Medicine Fellow

Department of Anesthesiology, Critical Care, and Pain Medicine

Beth Israel Deaconess Medical Center

Boston, Massachusetts

330 Brookline Avenue

Boston, MA 02115

E-mail: oviswana@bidmc.harvard.edu

Julie Petro, MD

Anesthesiologist/Interventional Pain Medicine Fellow

Department of Anesthesiology, Critical Care, and Pain Medicine

Beth Israel Deaconess Medical Center

330 Brookline Avenue

Boston, MA 02115

E-mail: jpetro@bidmc.harvard.edu

Abbas Asgerally, MD

Anesthesiologist/Interventional Pain Medicine Fellow

Department of Anesthesiology, Critical Care, and Pain Medicine

Beth Israel Deaconess Medical Center

330 Brookline Avenue

Boston, MA 02115

E-mail: aasgeral@bidmc.harvard.edu

Thomas T. Simopoulos, MD

Anesthesiologist, Interventional Pain Physician

Division Chair, Arnold-Warfield Pain Center

Department of Anesthesiology, Critical Care, and Pain Medicine

Beth Israel Deaconess Medical Center

330 Brookline Avenue

Boston, MA 02115

E-mail: tsimopou@bidmc.harvard.edu 


\section{References}

1. CNN Press Release: The More Opioids Doctors Prescribe, the More Money They Make. March 12, 2018.

2. Krebs EE, Gravely A, Nugent $S$, Jensen AC, DeRonne B, Goldsmith ES, Kroenke K, Bair MJ, Noorbaloochi S. Effect of opi- oid vs nonopioid medications on painrelated function in patients with chronic back pain or hip or knee osteoarthritis pain the SPACE randomized clinical trial. JAMA 2018; 319:872-882.

3. Jackson Healthcare: Physician Compen- sation Eight Percent Healthcare Costs. www.jacksonhealthcare.com/mediaroom/news/md-salaries-as-percent-ofcosts/ 